
\title{
28 Research Suare \\ Fracture Resistance of Primary Canines Restorations Reinforced with a Flowable or a Packable Composite Resin Posts; In- Vitro
}

\section{Ghasem Ansari}

Shahid Beheshti University of Medical Sciences School of Dentistry

\section{Fatemeh javadi}

Shahid Beheshti University of Medical Sciences School of Dentistry

\section{Kiana Hanjani}

Shahid Beheshti University of Medical Sciences School of Dentistry https://orcid.org/0000-0002-3393477X

\author{
Ahmad Najafi Abrandabadi \\ Shahid Beheshti University of Medical Sciences School of Dentistry \\ Mahshid Gashtasb \\ Shahid Beheshti University of Medical Sciences School of Dentistry \\ Romina Roodgarian ( $\nabla$ rominaroodgarian@gmail.com ) \\ School of Dentistry Shahid Beheshti University of Medical Sciences https://orcid.org/0000-0002-5777- \\ 6753
}

\section{Research}

Keywords: Fracture resistance, anterior, primary teeth, Composite resin, post

Posted Date: December 28th, 2021

DOI: https://doi.org/10.21203/rs.3.rs-1153008/v1

License: (a) (1) This work is licensed under a Creative Commons Attribution 4.0 International License. Read Full License 


\section{Abstract}

\section{Aim and Background:}

Restoration of severely damaged primary anterior teeth are deemed as serious challenges for pediatric dentists. The aim of this study was to evaluate the fracture resistance of primary canines restored with flowable or packable composite resin posts.

\section{Material and Methods}

This study was conducted on a group of extracted primary canines. After cutting the coronal section, standard pulpectomy was performed and root canals were filled with ZOE paste leaving $3 \mathrm{~mm}$ coronal root space. A liner was placed above the root canal filling as a barrier. Samples were then randomly divided into two groups of fifteen. Both groups, received etch, bond, then in group I Core Flo Composite resin placement into canal, in group II, post shaping using designated packable composite resin and inserted into the canal. Followed by Core buildup and Crown reconstruction using Z250 composite resin. Samples were mounted at $148^{\circ}$ angle on the acrylic blocks and stored in de-ionized water for 72 hours. Fracture resistance was measured using Universal Testing Machine. Data analysis were performed using independent t-test. Chi- square test were also employed to assess the type of fracture within groups.

\section{Results}

The mean fracture resistance in the group I was $830.31(+/-328.69 \mathrm{~N})$ and $803.09(+/-326.45 \mathrm{~N})$ in group II. Moreover, statistical analyses of the results showed no significant difference between the mean values of the fracture resistance $(p$-value $=0.822)$ of groups as well as the type of fracture $(p$-value $=0.79)$ between groups.

\section{Conclusion}

The ease of use and proper adhesion of the Core Flo composite resin, seems to help its use in the reconstruction of severely destructed anterior primary teeth.

\section{Introduction}

Dental caries is considered as the most common chronic and destructive dental disease during childhood which along with dental trauma, are the main causes for child referral for tooth restoration ${ }^{1}$ Nowadays, the prevalence of Early Childhood Caries (ECC) cases is growing remarkably, so that, children might face with several issues including, speech difficulties, reduction in facial vertical height and lack of confidence 
due to aesthetic concerns. Therefore, to avoid such problems, destructed teeth need to be restored properly ${ }^{2}$.

Restoration and maintenance of damaged primary teeth are challenging for pediatric dentists, as managing children behavior during the procedure is difficult ${ }^{2}$. In recent decades, using new generation of restorative materials introduced to the market such as strip crown, polycarbonate crown, veneered stainless steel crowns, and ART glass, it is feasible to restore decayed teeth with an inadequate structure

${ }^{3}$ However, the application of these materials could lead to failure due to their intolerance toward intensive occlusal forces ${ }^{4,5}$ Despite of numerous issues existing in the usage of composite resin, these substances recommended as the materials of choice for restoration of anterior teeth because of aesthetic demands ${ }^{6}$.

If the anterior tooth is significantly destructed, only a small fraction of tooth and its enamel remains for bonding. This is due to the fact that it has a narrow crown. In addition, the residual enamel of the cervical area of tooth has a restricted capability for bonding to restorative material ${ }^{1}$ To overcome this issue, it has been suggested to use intracanal post or similar retainers following pulpectomy of tooth in order to enforce retention of coronal restoration 5

Presently, numerous types of posts are used in pediatric dentistry like, Nickle -Titanium posts, orthodontic wires, biologic posts, composite posts, fiber reinforced composite, fiber-based posts and ceramic posts. $^{7-9}$

One of the simplest and most-effective approaches recommended for the restoration of significantly destroyed primary anterior teeth is to pack the composite resin into the canal and construct a short composite resin post 10 Composite resin posts have a similar modulus of elasticity with intracanal dentin and will easily bond to the tooth structure. As a result of an adequate mechanical retention, a better distribution of occlusal forces exists with these posts. Simple application, no necessity to laboratory fabrication, low price, and optimal adaptation are among the other advantages of composite resin posts. 9-11

Composite resin posts are efficient as there is adequate homogeneity between them and covering composite resin. In addition, contrary to metallic post, it is not necessary to apply an opaque layer of composite resin to obscure metal. On the other hand, regarding polymerization shrinkage and reduction in retention, short composite posts are more prone to fracture ${ }^{9}$. Having adequate fracture resistance is important while using this method to restore primary teeth. This means an inadequate fracture resistance can lead to breakage of material under the pressure. The type of restorative material and the amount of tooth structure that has been lost are two important factors in this phenomenon. ${ }^{8}$

The aim of this investigation was to evaluate the fracture resistance of primary anterior canine restorations reinforced with either a flowable or a packable composite resin post.

\section{Method And Materials}


Study protocol was approved by the Ethics Committee of the Dental School at Shahid Beheshti University of Medical Sciences. Thirty healthy freshly extracted canine teeth were obtained with at least $2 / 3$ root length remaining. The teeth with any degrees of caries, previous restorations and visible fractures or cracks were excluded from this study. Parents of patients were informed of the purpose of this study too. Dental calculus and residual soft tissues were removed from tooth surface by dental scalers and curettes. All teeth were placed in $0.5 \%$ chloramine solution for a week and then stored in distilled water at $4{ }^{\circ} \mathrm{C}$ before test course. The teeth were then cut from the coronal section and $1 \mathrm{~mm}$ above the CEJ by a diamond bur on a high speed hand piece with air and water spray. The pulp was then removed through a standard pulpectomy procedure. Subsequently, root canals were filled with ZOE paste (PD, Switzerland).A 48 hours period was taken to complete the pulpectomy process after which $4 \mathrm{~mm}$ of ZOE paste was removed from the coronal part of the canal using a slow-speed hand piece with a round carbide bur. A 1 $\mathrm{mm}$ thick of liner (Pulpdent, USA) was then placed on top of the ZOE paste and before placement of composites resin posts.

Samples were then randomly divided into two groups of 15 .

In the group I, flowable composite (Core Flo DC LITE, USA) being used to make the post while the restoration of the crown was performed with a conventional packable composite (composite 3M EPSE Z250, USA).In the group II, packable composite(3M EPSE Z250, USA) was used to make the post along with the final restoration. Root canals and residual enamel were etched with $37 \%$ Phosphoric Acid gel (Scothbond ${ }^{\mathrm{TM}}$ Etchant, 3M ESPE, MN, USA) for 15 seconds, rinsed for 30 seconds and dried for 10 seconds, whereas the dentin area remained slightly moist. Thereafter, two consecutive coats of bonding agent (Tetric-N Bond, Ivocolar Vivadent, Switzerland) were applied on the etched surfaces, uniformly dispersed by a compressed air blast for 2-5 seconds and cured by an LED light cure (Woodpecker, China) for 20 seconds. Finally, the composite resin was placed in layers with a thickness of $1.5 \mathrm{~mm}$ in the root canal space with each layer being subjected to curing for 40 seconds. Posts were built to $2 \mathrm{~mm}$ above the orifice and the crowns were built up with an approximate height of $4 \mathrm{~mm}$ (normal size).

Each tooth was then placed in acrylic blocks from the apical portion ( $2 \mathrm{~mm}$ below CEJ) and mounted at $148^{\circ}$ angle (Acrylic acrylic, Acropars, Iran).

All sample teeth were floated in de-ionized water for about 72 hours, at room temperature. In order to evaluate fracture resistance under regulated pressure, they have been loaded by Universal Testing Machine (Zwick Role, Denmark).

Samples were fixed in a special fixture and received a progressively increasing load with a crosshead speed of $0.5 \mathrm{~mm} / \mathrm{min}$ along the long axis of the primary canines on the mid-palatal surface. The increase in force was continued until the specimen was fractured. The pattern of fracture was then looked at using a 10x magnification stereomicroscope. Pattern of failure on samples were as follows:

1) Adhesion failure: Failure in the adhesive-tooth interfaces or the adhesive-composite interfaces. 
2a) cohesive failure of resin: This failure occurs mainly in composites.

2b) cohesive failure of the tooth: This failure occurs mainly in the teeth.

3. Mixed Failure: A mixture of the above failures is observed.

Independent t-test was used to compare the fracture resistance in two methods of tooth reconstruction. Pvalue of $<0.05$ was considered as a significant limit. Chi-square test was used to determine the differences between the types of failure of the two groups.

\section{Results}

Minimum, maximum and the mean of fracture resistance in two groups were recorded and compared (Table 1). Independent t-test was conducted to compare fracture resistance between the two groups. The mean value of fracture resistance in group I (Reinforced with Core Flo composite resin post and core) was higher than group II (reinforced with packable composite resin post and core), however this difference was not statistically significant ( $p$ - value $=0.82$ ).

\section{Table1}

Fracture resistance in two groups in Newton (N)

\begin{tabular}{|llllllll|}
\hline Group & composite post & Quantity & Min FR & Max FR & Mean & SD & p-value \\
\hline I & Core Flo & 15 & 426.72 & 1580 & 830.31 & 328.69 & 0.822 \\
II & Packable & 15 & 337.78 & 1164.84 & 770.51 & 326.45 & \\
\cline { 1 - 5 } & & & &
\end{tabular}

The type of fracture was evaluated under stereo microscope (Table 2).

Chi- square test showed no significant difference between fracture resistance of the two groups ( $p$ value $=0.79$ ).

Table 2

Frequency of fractures in group I and II

\begin{tabular}{|llllll|}
\hline \multirow{2}{*}{ composite post } & \multicolumn{3}{l}{ Type of fracture } & \\
\cline { 2 - 6 } & & Adhesive & Cohesive & Mixed & Cohesive failure of tooth \\
\hline \multirow{2}{*}{ Core Flo } & Number & 0 & 14 & 1 & 0 \\
\cline { 2 - 6 } & Percentage & 0 & 93.33 & 6.67 & 0 \\
\hline \multirow{2}{*}{ Packable } & Number & 2 & 10 & 3 & 0 \\
\cline { 2 - 6 } & percentage & 13.33 & 66.67 & 20 & 0 \\
\hline \multirow{2}{*}{ Total number } & 2 & 24 & 4 & 0 \\
\hline
\end{tabular}




\section{Discussion}

During daily routine activities, teeth are being impacted by various mechanical forces in the oral cavity. These forces directly affect the durability of teeth restorations. Different tests have been conducted to analyze the mechanical forces applied on teeth including shear bond strength and fracture resistance. Each of these tests has been used to assess those forces applied on teeth. Enhancement of physical characteristics of restorative materials is considered as a critical priority when applying any restorative material on teeth. ${ }^{12}$ Fracture resistance is considered as one of the main features of a suitable restorative material and one of the critical factors in attaining durable restorations ${ }^{13}$.

Findings of the current investigation revealed that the mean of forces leading to fracture in two groups as $830 \mathrm{~N}$ (Newton) and 808N (Newton) respectively, similar to study of Rentus et al. which was conducted on children between 3 to 5.5 years old with different types of occlusion. The results of Mountain et al. study revealed that $t$ the maximum bite force at the first and second molars and central incisors ranged from 12.61 to $353.6 \mathrm{~N}$ (mean $196.6 \mathrm{~N}$ ) ${ }^{14,15}$. The difference between current study result and their results could be due to the nature on their in vivo study while current one was in vitro. However, such observations could provide information on the integrity of tooth and restoration demonstrating the weak structural part of the samples ${ }^{16}$.

Kadkhodaei et al. stated that the fracture resistance of anterior teeth restored with Z250 composite resin without intracanal post were at $296.58 \pm 98.35$ whereas in the present study this number was in range between 808 to $803 \mathrm{~N}$ (Newton). This difference could indicate the value of such composite resin posts 17,18 .

Earlier studies have argued that the materials applied as a short post and core in anterior primary Maxillary teeth are more functional as these materials have closer elasticity to that of dentine. ideal intracanal post provides adequate retention while are with appropriate facture resistance proof too. Similarity of the post shape and shape of the canal provide a higher level of distribution of forces along the coronal restoration while reducing probability of tooth fracture [13]. Among different types of introduced approaches, compacting composite into the canal space in order to construct a short post has been propounded as the best option ${ }^{18}$.Using composite posts are preferred due to their ease of use, low cost, no laboratory step, and good versatility. Wide use of composites in dental clinics is an indication of the need to carry more specificity studies on them ${ }^{9}$.

Malakar et al. demonstrated that in spite of highest fracture resistance among composite in their studied groups, the statistical analysis revealed no significant difference. As a result, all the three types of post including composite resin, glass fiber posts and, ribbond posts, were concluded to be used to reconstruct severely damaged primary anterior teeth ${ }^{19}$.

Moreover, according to results of study which has been conducted by Fallahinejad Ghajari et al. the Grandio Flow composite core and Grandio Flow post revealed the highest fracture resistance 
(474.7 \pm 100.8 N) while Grandio composite restorative core and Grandio composite restorative post demonstrated the lowest fracture resistance $(364.4 \pm 74.5 \mathrm{~N})$. Although, the fracture resistance between two studied groups showed no significant difference, similar to the current findings ${ }^{20}$.

Furthermore, Seraj et al. demonstrated that, the difference of fracture strength, fracture resistance and, fracture mode of composite resin was not significant between resin composite post, prefabricated glass fiber and customized quartz fiber post ${ }^{21}$.

Similar diameter anterior teeth at CEJ were included in this investigation in order to match the sample sizes for similar crown reconstruction bulks. The clinical success of post and core restoration depends on the type of composite and the quality of adhesion between post and core, the area that materials with different combination are in relation with each other. The durability of restoration with composite resin core rely in the bond strength between composite resin and remaining tooth structure and the bond strength between the composite resin and post ${ }^{22}$. The differences in fracture types between the two groups were statistically insignificant in the current investigation.

Pithan et al. concluded that no significant difference could be seen between composite resin posts, orthodontic wires and fiber glass posts. The application of orthodontic wire as an intra-canal retention was reported as a more technique sensitive in comparison to the posts made of composite resin. In addition, orthodontic wires could lead to root fracture while tooth is exposed to intensive mastication forces ${ }^{23}$.

Sadek et al. stated that bond strength of various types of composite resin used as core material adjacent to fiber posts was highest in Core-Flo (bisco). Although, the difference between groups was not statistically significant ${ }^{24}$.Clinical application of flowable composite resin for reconstruction of severely destroyed teeth because of its inherent weakness, low modulus of elasticity and inadequate strength toward occlusal forces is questionable. It seems that concurrent utilization of adaptive properties of flowable composite resin used as core material could serve as an acceptable restoration for root treated teeth 24,25

\section{Conclusion}

According to the results of this investigation with the high adhesion potential of the Core Flo composite, it appears that restored teeth with this material have a higher resistance rate to fracture of the restored anterior primary teeth.

\section{Declarations}

\section{Conflict of Interest:}

No conflict of interest to declare. 


\section{Ethical Approval and Consent to participate}

None to Declare.

\section{Consent for publication}

We have full consent for free publication.

\section{Availability of data and materials}

The data that support the findings of this study are available from Deparment of pedodontics of shahid beheshti university of medical sciences, but restrictions apply to the availability of these data, which were used under license for the current study, and so are not publicly available. Data are however available from the authors upon reasonable request and with permission of Deparment of pedodontics of shahid beheshti university of medical sciences.

\section{Competing interests}

None to Declare.

\section{Funding}

None to declare.

\section{Authors Contributions}

Dr.Hanjani collected the data and wrote the manuscript. Dr.Roodgarian collected the data and helped writing the manuscript. Dr.Najafi performed the statistical analysis. Dr.Gashtasb supervised the data collection and manuscript writing. Dr.Ghasemi supervised the whole study from data collection, statistical analysis, and manuscript writing. The author(s) read and approved the final manuscript.

\section{Acknowledgement:}

Authors would like to acknowledge the tremendous help of the research committee of SBMU Dental School including their statistician expert.

\section{Authorsinformation}

Ghassem Ansari ${ }^{1}$, Fatemeh Javadi ${ }^{2}$, Kiana Hanjani ${ }^{3}$, Ahmad Najafi Abrandabadi ${ }^{4}$, Mahshid Gashtasb $^{5}$, Romina Roodgarian ${ }^{3}$

${ }^{1}$ Professor, Department of Pediatric Dentistry\& Research Institute for Dental Sciences, School of Dentistry Shahid Beheshti University of Medical Sciences, Tehran, Iran

2 Private pedodontist, Tehran, lan 
${ }^{3}$ Postgraduate Student, Department of Pediatric Dentistry, School of Dentistry Shahid Beheshti University of Medical Sciences, Tehran, Iran

${ }^{4}$ Faculty Member, Department of Restorative Dentistry, School of Dentistry Shahid Beheshti university of Medical Sciences, Tehran, Iran

${ }^{5}$ Graduate Student, Research Institute of Dental Sciences, Dental school, Shahid Beheshti University of Medical Sciences, Tehran, Iran

Corresponding Author: Romina Roodgarian,

Department of Pediatric Dentistry,

Dental School Shahid Beheshti University of Medical Sciences,

Tehran, Iran

E-mail: rominaroodgarian@gmail.com

Corresponding author

Correspondence to Dr.Romina roodgarian.

\section{References}

1. Talebi M, Parisay I, Khorakian F, Nik E. A Simplified Method for the Restoration of Severely Decayed Primary Incisors. Journal of Dentistry (Tehran, Iran). 2015;12(3):177.

2. Usha M, Deepak V, Venkat S, Gargi M. Treatment of severely mutilated incisors: a challenge to the pedodontist. Journal of Indian Society of Pedodontics and Preventive Dentistry. 2007;25(5):34.

3. Ram D, Peretz B. Composite crown-form crowns for severely decayed primary molars: a technique for restoring function and esthetics. Journal of Clinical Pediatric Dentistry. 2000;24(4):257-260.

4. Sharaf A. The application of fiber core posts in restoring badly destroyed primary incisors. Journal of Clinical Pediatric Dentistry. 2002;26(3):217-224.

5. Memarpour M, Shafiei F, Abbaszadeh M. Retentive strength of different intracanal posts in restorations of anterior primary teeth: an in vitro study. Restorative dentistry \& endodontics. 2013;38(4):215-221.

6. Nilavarasan N, Hemalatha R, Vijayakumar R, Hariharan V. Comparison of compressive strength among three different intracanal post materials in primary anterior teeth: An in vitro study. European journal of dentistry. 2016;10(4):464.

7. Mortada A, King N. A simplified technique for the restoration of severely mutilated primary anterior teeth. Journal of Clinical Pediatric Dentistry. 2004;28(3):187-192. 
8. Grewal N, Seth R. Comparative in vivo evaluation of restoring severely mutilated primary anterior teeth with biological post and crown preparation and reinforced composite restoration. Journal of Indian Society of Pedodontics and Preventive Dentistry. 2008;26(4):141.

9. Judd PL, Kenny DJ, Johnston DH, Yacobi R. Composite resin short-post technique for primary anterior teeth. The Journal of the American Dental Association. 1990;120(5):553-555.

10. Donly KJ, García-Godoy F. The use of resin-based composite in children: an update. Pediatric Dentistry. 2015;37(2):136-143.

11. Paryab M, Afshar H, Seraj B, Shakibapoor S, Kharazifard MJ. Fracture strength of severely damaged primary anterior teeth after restoration with composite resin and resin-modified glass ionomer cement. Journal of Islamic Dental Association of Iran. 2016;28(2):57-63.

12. Newman MP, Yaman P, Dennison J, Rafter M, Billy E. Fracture resistance of endodontically treated teeth restored with composite posts. The Journal of prosthetic dentistry. 2003;89(4):360-367.

13. Gurbuz T, Sengul F, Altun C. Finite element stress analysis of short-post core and over restorations prepared with different restorative materials. Dental materials journal. 2008;27(4):499-507.

14. Rentes A, Gavião M, Amaral J. Bite force determination in children with primary dentition. Journal of oral rehabilitation. 2002;29(12):1174-1180.

15. Mountain G, Wood D, Toumba J. Bite force measurement in children with primary dentition. International journal of paediatric dentistry. 2011;21(2):112-118.

16. Alavi A, Zahedi S. Evaluation of fracture resistance of teeth restored with three types of tooth colored onlay. Journal of Mashhad Dental School. 2006;30(Issue):289-300.

17. Kadkhodaei F, Mehran M, Haghgoo R, Zareiyan M. Fracture Resistance of Three Post Types in the Restoration of Anterior Primary Teeth. The Open Dentistry Journal. 2020;14(1).

18. Ferreira M, Kummer T, Vieira R, Calvo M. Short Resin-posts Bonding to Primary Dentin. Microleakage and Micro-morphological an in vitro study. Journal of Clinical Pediatric Dentistry. 2007;31(3):202206.

19. Malakar S, Tripathi S, Rahat A. Analysis of Fracture Resistance of Different Posts in Restoration of Severely Damaged Primary Anterior Teeth: An In Vitro Study. 2019.

20. Fallahinejad Ghajari M, Majidi Rad H, Baghalian A. Fracture Resistance of Maxillary Primary Anterior Restorations Using Grandio and Grandio Flow Composites with Two Differ-ent Types of Posts: An In Vitro Study. Journal of Islamic Dental Association of Iran. 2019;31(3):139-145.

21. Seraj B, Ghadimi S, Estaki Z, Fatemi M. Fracture resistance of three different posts in restoration of severely damaged primary anterior teeth: An in vitro study. Dental research journal. 2015;12(4):372.

22. Quintas AF, Bottino MA, Neisser MP, Araújo MAJd. Effect of the surface treatment of plain carbon fiber posts on the retention of the composite core: an in vitro evaluation. Pesquisa Odontológica Brasileira. 2001;15(1):64-69.

23. Pithan S, de Sousa Vieira R, Chain MC. Tensile bond strength of intracanal posts in primary anterior teeth: an in vitro study. Journal of Clinical Pediatric Dentistry. 2003;27(1):35-39. 
24. Sadek FT, Monticelli F, Goracci C, Tay FR, Cardoso PE, Ferrari M. Bond strength performance of different resin composites used as core materials around fiber posts. Dental Materials. 2007;23(1):95-99.

25. Radovic I, Monticelli F, Goracci C, et al. The effect of sandblasting on adhesion of a dual-cured resin composite to methacrylic fiber posts: microtensile bond strength and SEM evaluation. journal of dentistry. 2007;35(6):496-502. 